03

\title{
Спектроскопия слоев на плоскопараллельных подложках
}

\author{
() А.Б. Сотский', С.С. Михеевㄹ , Н.И. Стаськов ${ }^{1}$, Л.И. Сотская² \\ ${ }^{1}$ Могилевский государственный университет им. А.А. Кулешова, \\ 212022 Могилев, Беларусь \\ ${ }^{2}$ Белорусско-Российский университет, \\ 212000 Могилев, Беларусь \\ e-mail: ab_sotsky@mail.ru
}

Поступила в редакцию 05.03.2020 г.

В окончательной редакции 05.03.2020 г.

Принята к публикации 28.03.2020 г.

Получены интегральные выражения для спектров отражательной и пропускательной способностей структуры в виде двух тонких слоев, нанесенных на противоположные грани плоскопараллельной подложки в условиях наклонного освещения структуры частично когерентным светом. В результате асимптотического анализа интегралов установлены приближенные аналитические формулы для расчета названных спектров, удобные для использования при решении обратных задач спектрофотометрии. Исследован допированный алюминием слой оксида цинка, нанесенный на стеклянную подложку. Спектры показателей преломления и поглощения слоя и подложки, а также толщина слоя восстановлены путем обработки спектров отражательной и пропускательной способностей структуры, измеренных для волн $s$ - и $p$-поляризации при двух углах падения света на структуру. Найденные параметры структуры использованы в вычислительных экспериментах для оценок границ применимости сформулированных приближений.

Ключевые слова: спектрофотометрия, частичная когерентность, обратная оптическая задача, отражательная и пропускательная способности.

DOI: $10.21883 /$ OS.2020.08.49711.79-20

\section{Введение}

При контроле геометрических и оптических характеристик слоев различных материалов широко применяется метод спектрофотометрии. Для большинства спектрофотометров характерно использование широкополосных источников света с выделением узкого участка длин волн

$$
\lambda_{0}-\Delta \lambda \leq \lambda \leq \lambda_{0}+\Delta \lambda \quad\left(\Delta \lambda / \lambda_{0} \ll 1\right)
$$

при помощи перестраиваемого монохроматора. В такой ситуации зондирующее слой излучение является частично когерентным. Эта особенность может оказывать существенное влияние на измеряемые спектры отражательной $R(\lambda)$ и пропускательной $T(\lambda)$ способностей исследуемой структуры. В частности, частичную когерентность излучения необходимо учитывать при решении обратных задач спектрофотометрии для слоев, расположенных на плоскопараллельных слабо поглощающих подложках [1-12].

В работах $[1,2,5,7,9-11]$ используются различные варианты феноменологического подхода, в котором в исследуемой плоскопараллельной структуре выделяются так называемые когерентные и некогерентные однородные слои [9]. Для когерентных слоев, толщина которых сопоставима с $\lambda$, коэффициенты отражения и пропускания света рассчитываются в рамках модели монохроматического света. Плоскопараллельная подложка с показателем преломления $n_{s}$ и толщиной $D \gg \lambda^{2}\left(2 n_{s} \Delta \lambda\right)^{-1}$ [7] считается некогерентным слоем.
Этот слой рассматривается в лучевом приближении, в котором лучи различного порядка, отраженные от его границ, считаются некогерентными. Итоговые выражения для $R(\lambda)$ и $T(\lambda)$ получаются суммированием рядов Эйри. В явном виде данные выражения достаточно громоздки и не всегда свободны от опечаток, как это отмечено в тщательно выверенной работе [11]. Основной же недостаток приближения когерентных и некогерентных слоев состоит в отсутствии его электродинамического обоснования и, как следствие, точных оценок границ его применимости в зависимости от $D$ и $\Delta \lambda$.

В $[3,4,6,12]$ с целью учета интерференции лучей различного порядка в окружающем структуру пространстве в рассмотрение различными способами вводится комплексная степень их когерентности $\gamma$. Однако появление двух дополнительных неизвестных в виде вещественной и мнимой частей $\gamma$ в зависимостях $R(\lambda)$ и $T(\lambda)[3,4,6,12]$ существенно усложняет решение обратных задач спектрофотометрии.

Более эффективный подход к решению проблемы частичной когерентности зондирующего света, не требующий введения степени когерентности $\gamma$, был предложен в [10]. Здесь зависимости $R(\lambda)$ и $T(\lambda)$ определяются как результат усреднения функций $|r(\lambda)|^{2}$ и $|t(\lambda)|^{2}(r(\lambda)$ и $t(\lambda)$ - амплитудные коэффициенты отражения и прохождения плоских волн) по спектральной плотности падающего на структуру излучения $\hat{\rho}(\lambda)$. В [10] получены аналитические выражения для $R(\lambda)$ и $T(\lambda)$. Однако данные выражения справедливы только в случае 
ступенчатой модели $\hat{\rho}(\lambda)$, которая для реальных спектрофотометров может быть достаточно грубой.

В случае наклонного падения света на плоскопараллельную структуру под углом $\theta$ ее собственные волны имеют $s$ - либо $p$-поляризацию. Поэтому далее в рассмотрение вводится индекс поляризации $\alpha(\alpha=1$ для волн $s$-поляризации, $\alpha=2$ для волн $p$-поляризации), и определенные выше функции $R(\lambda), T(\lambda),|r(\lambda)|^{2},|t(\lambda)|^{2}$ заменяются на $R_{\alpha}(\theta, \lambda), T_{\alpha}(\theta, \lambda),\left|r_{\alpha}(\theta, \lambda)\right|^{2},\left|t_{\alpha}(\theta, \lambda)\right|^{2}$.

В настоящей работе спектры $R_{\alpha}(\theta, \lambda)$ и $T_{\alpha}(\theta, \lambda)$ рассчитываются с учетом частичной когерентности излучения путем интегрирования распределений $\left|r_{\alpha}(\theta, \lambda)\right|^{2}$ и $\left|t_{\alpha}(\theta, \lambda)\right|^{2}$ в произведении с аппаратной функцией спектрофотометра. При выполнении характерного для реальных структур условия $D \Delta \lambda \gg \lambda^{2}$ подынтегральные функции соответствующих интегралов быстро осциллируют, что затрудняет вычисление интегралов и их использование при решении обратных задач спектрофотометрии, которые требуют десятков миллионов обращений к расчету отражательной и пропускательной способностей структуры. С целью упрощения вычислений в первом разделе работы выполнен асимптотический анализ данных интегралов при $D \rightarrow \infty$ и $D \rightarrow 0$. В результате получены приближенные аналитические формулы для $R_{\alpha}(\theta, \lambda)$ и $T_{\alpha}(\theta, \lambda)$, которые не зависят от вида аппаратной функции спектрофотометра. Во втором разделе данные формулы применены к решению обратной задачи спектрофотометрии для наноразмерного слоя $\mathrm{ZnO}: \mathrm{Al}$, расположенного на плоскопараллельной стеклянной подложке. Обработка зависимостей $R_{\alpha}(\theta, \lambda)$ и $T_{\alpha}(\theta, \lambda)$, измеренных при двух углах падения излучения на структуру, выполнена с позиций аналитического определения дисперсионных характеристик подложки и численного решения обратной задачи спектрофотометрии однородного слоя на подложке. Спектры показателя преломления $n_{f}(\lambda)$, поглощения $k_{f}(\lambda)$ слоя и его толщина $d$ определены методом наименьших квадратов с использованием представления комплексного показателя преломления слоя полиномом Лагранжа-Чебышева оптимальной степени. Найденные значение $d$ и функции $n_{f}(\lambda), k_{f}(\lambda)$ использованы для оценки границ применимости аналитических формул для $R_{\alpha}(\theta, \lambda)$ и $T_{\alpha}(\theta, \lambda)$ в случаях гауссовой и прямоугольной моделей аппаратной функции спектрофотометра.

\section{Расчет функций $R_{\alpha}(\theta, \lambda)$ и $T_{\alpha}(\theta, \lambda)$}

Исследуемая плоскопараллельная структура представлена на рис. 1. В областях $1(y>0)$ и 5 $\left(y<-d-D-d^{\prime}\right)$ находится воздух с показателем преломления $n_{a}$. Подложка 3 с показателями преломления и поглощения $n_{s}$ и $k_{s}$ занимает область $-D-d \leq y \leq-d$. На противоположных гранях подложки находятся в общем случае неоднородные слои 2 и 4 с толщинами $d^{\prime}$ и $d$. Структура возбуждается плоской волной $s$ - либо $p$-поляризации, падающей из области $y>0$ под углом $\theta$.

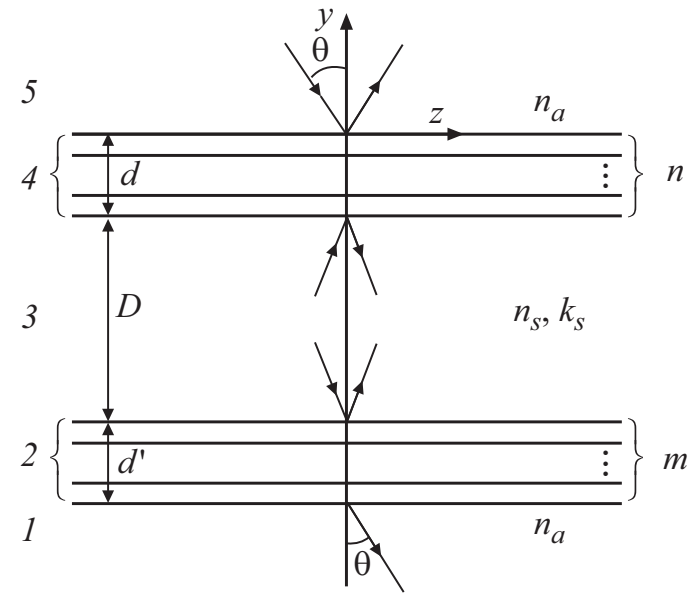

Рис. 1. Слои на плоскопараллельной подложке.

Компоненты электрического поля $E_{i}^{(\alpha)}(t), E_{r}^{(\alpha)}(t)$ и $E_{t}^{(\alpha)}(t)$ на фронтах падающей, отраженной и прошедшей волн соответственно предполагаются стохастическими функциями времени, представимыми интегралами Фурье

$$
E_{i, r, t}^{(\alpha)}(t)=\int_{-\infty}^{\infty} \hat{E}_{i, r, t}^{(\alpha)}(\omega) \exp (i \omega t) d \omega
$$

При наличии в схеме спектрофотометра монохроматора имеют место соотношения

$$
\begin{gathered}
\hat{E}_{i}^{(\alpha)}(\omega)=g_{\alpha} \hat{E}_{0}^{(\alpha)}(\omega), \quad \hat{E}_{r}^{(\alpha)}(\omega)=r_{\alpha}(\theta, \lambda) g_{\alpha}(\omega) \hat{E}_{0}^{(\alpha)}(\omega), \\
\hat{E}_{r}^{(\alpha)}(\omega)=t_{\alpha}(\theta, \lambda) g_{\alpha}(\omega) \hat{E}_{0}^{(\alpha)}(\omega),
\end{gathered}
$$

где $\hat{E}_{0}^{\alpha}(\omega)$ - фурье-образ напряженности поля $E_{0}^{\alpha}(t)$, создаваемого источником излучения, $g_{\alpha}(\omega)-$ комплексный коэффициент пропускания монохроматора, $\lambda=2 \pi c \omega^{-1}, c-$ скорость света. При измерении функций $E_{i}^{\alpha}(t), E_{r}^{\alpha}(t)$ либо $E_{t}^{\alpha}(t)$ на фотоприемнике спектрофотометра формируется сигнал, пропорциональный $\left\langle\left[E_{i}^{\alpha}(t)\right]^{2}\right\rangle,\left\langle\left[E_{r}^{\alpha}(t)\right]^{2}\right\rangle$ либо $\left\langle\left[E_{t}^{\alpha}(t)\right]^{2}\right\rangle$, где угловые скобки означают усреднение стоящих в них величин по времени [13]. Предполагая процесс излучения света источником стационарным и эргодическим, заменяем указанное временное усреднение усреднением по ансамблю процессов:

$$
\begin{aligned}
& \left\langle\left[E_{i}^{(\alpha)}(t)\right]^{2}\right\rangle=\frac{1}{(2 \pi)^{2}} \int_{-\infty}^{\infty} d \omega_{1} g_{\alpha}\left(\omega_{1}\right) \int_{-\infty}^{\infty} d \omega_{2} g_{\alpha}\left(\omega_{2}\right) \\
& \times \int_{-\infty}^{\infty} \int_{-\infty}^{\infty} d t_{1} d t_{2} \exp \left[i \omega_{1}\left(t-t_{1}\right)+i \omega_{2}\left(t-t_{2}\right)\right] \overline{E_{0}^{(\alpha)}\left(t_{1}\right) E_{0}^{(\alpha)}\left(t_{2}\right)},
\end{aligned}
$$




$$
\begin{aligned}
\left\langle\left[E_{r}^{(\alpha)}(t)\right]^{2}\right\rangle= & \frac{1}{(2 \pi)^{2}} \int_{-\infty}^{\infty} d \omega_{1} g_{\alpha}\left(\omega_{1}\right) r_{\alpha}\left(\theta, \lambda_{1}\right) \\
& \times \int_{-\infty}^{\infty} d \omega_{2} g_{\alpha}\left(\omega_{2}\right) r_{\alpha}\left(\theta, \lambda_{2}\right) \\
& \times \int_{-\infty}^{\infty} \int_{-\infty}^{\infty} d t_{1} d t_{2} \exp \left[i \omega_{1}\left(t-t_{1}\right)\right. \\
& \left.+i \omega_{2}\left(t-t_{2}\right)\right] \overline{E_{0}^{(\alpha)}\left(t_{1}\right) E_{0}^{(\alpha)}\left(t_{2}\right)} \\
\left\langle\left[E_{t}^{(\alpha)}(t)\right]^{2}\right\rangle= & \frac{1}{(2 \pi)^{2}} \int_{-\infty}^{\infty} d \omega_{1} g_{\alpha}\left(\omega_{1}\right) t_{\alpha}\left(\theta, \lambda_{1}\right) \\
& \times \int_{-\infty}^{\infty} d \omega_{2} g_{\alpha}\left(\omega_{2}\right) t_{\alpha}\left(\theta, \lambda_{2}\right) \\
& \times \int_{-\infty}^{\infty} \int_{-\infty}^{\infty} d t_{1} d t_{2} \exp \left[i \omega_{1}\left(t-t_{1}\right)\right. \\
& \left.+i \omega_{2}\left(t-t_{2}\right)\right] \overline{E_{0}^{(\alpha)}\left(t_{1}\right) E_{0}^{(\alpha)}\left(t_{2}\right)}
\end{aligned}
$$

где черта означает усреднение по ансамблю, $\lambda_{1,2}=2 \pi c \omega_{1,2}^{-1}$. При получении (4)-(6) выполнено обратное преобразование Фурье в (2), и в аналогичном выражении для $E_{0}^{\alpha}(t)$ учтены формулы (3). Вводя в рассмотрение автокорреляционную функцию источника $\rho_{\alpha}(\tau)=\overline{E_{0}^{\alpha}(t) E_{0}^{\alpha}(t+\tau)}$, принимая во внимание ее четность и замечая, что вследствие вещественности реального оптического поля $g_{\alpha}(-\omega)=g_{\alpha}^{*}(\omega)$, $r_{\alpha}(\theta,-\lambda)=r_{\alpha}^{*}(\theta, \lambda), \quad t_{\alpha}(\theta,-\lambda)=t_{\alpha}^{*}(\theta, \lambda) \quad$ (звездочка означает комплексное сопряжение), из (4) получаем

$$
\left\langle\left[E_{t}^{(\alpha)}(t)\right]^{2}\right\rangle=\int_{-\infty}^{\infty}\left|g_{\alpha}(\omega)\right|^{2} \hat{\rho}_{\alpha}(\omega) d \omega .
$$

Здесь

$$
\hat{\rho}_{\alpha}(\omega)=(2 \pi)^{-1} \int_{-\infty}^{\infty} \rho_{\alpha}(\tau) \cos (\omega \tau) d \tau
$$

- спектральная плотность излучения источника света [14],

$$
\left|g_{\alpha}(\omega)\right|^{2}=G_{\alpha}\left[\left(\omega-\omega_{0}\right)(\Delta \omega)^{-1}\right]+G_{\alpha}\left[\left(\omega+\omega_{0}\right)(\Delta \omega)^{-1}\right],
$$

$\omega_{0}=2 \pi c \lambda_{0}^{-1}>0, \Delta \omega / \omega_{0}=\Delta \lambda / \lambda_{0}, G_{\alpha}(x)$ имеет смысл аппаратной функции спектрофотометра, которая для реальных приборов существенно отличается от нуля при $|x| \leq 1$ и является четной [15].

Допустим, что функция $\hat{\rho}_{\alpha}(\omega)$ пренебрежимо мало изменяется на интервале $\omega_{0}-\Delta \omega \leq \omega \leq \omega_{0}+\Delta \omega$. То- гда (7) можно записать в виде

$$
\left\langle\left[E_{i}^{\alpha}(t)\right]^{2}\right\rangle=2 \hat{\rho}_{\alpha}\left(\omega_{0}\right) \Delta \omega \int_{-\infty}^{\infty} G_{\alpha}(x) d x .
$$

Проводя аналогичные преобразования в (5) и (6), приходим к выражениям

$$
\begin{aligned}
R_{\alpha}\left(\theta, \lambda_{0}\right)= & \left\langle\left[E_{r}^{(\alpha)}(t)\right]^{2}\right\rangle\left\langle\left[E_{i}^{(\alpha)}(t)\right]^{2}\right\rangle^{-1} \\
& =\frac{\int_{-\infty}^{\infty} G_{\alpha}\left[\left(\omega-\omega_{0}\right)(\Delta \omega)^{-1}\right]\left|r_{\alpha}(\theta, \lambda)\right|^{2} d \omega}{\Delta \omega \int_{-\infty}^{\infty} G_{\alpha}(x) d x}, \\
T_{\alpha}\left(\theta, \lambda_{0}\right)= & \left\langle\left[E_{t}^{(\alpha)}(t)\right]^{2}\right\rangle\left\langle\left[E_{i}^{(\alpha)}(t)\right]^{2}\right\rangle^{-1} \\
= & \frac{\int_{-\infty}^{\infty} G_{\alpha}\left[\left(\omega-\omega_{0}\right)(\Delta \omega)^{-1}\right]\left|t_{\alpha}(\theta, \lambda)\right|^{2} d \omega}{\Delta \omega \int_{-\infty}^{\infty} G_{\alpha}(x) d x} .
\end{aligned}
$$

Конкретизируем функции $r_{\alpha}(\theta, \lambda)$ и $t_{\alpha}(\theta, \lambda)$. Для волн $s$-поляризации $x$-компонента электрического поля, равная $u$, описывается системой обыкновенных дифференциальных уравнений [16]

$$
d u / d y=v, \quad d v / d y=-k_{0}^{2}\left[\varepsilon(y)-\varepsilon_{\alpha} \sin ^{2} \theta\right] u,
$$

где $k_{0}=\omega c^{-1}-$ волновое число вакуума, $\varepsilon(y)-$ диэлектрическая проницаемость слоистой среды, $\varepsilon_{a}=n_{a}^{2}$. В окрестности слоя 4 , занимающего область $-d<y<0$ (рис. 1), общее решение системы (10) равно сумме его двух частных решений с произвольными амплитудами. Пусть первое из этих решений $\left(u=u_{1}(y), v=v_{1}(y)\right)$ описывается выражениями

$$
\begin{gathered}
u_{1}=a_{5}^{(1)} \exp \left(i k_{y a} y\right)+b_{5}^{(1)} \exp \left(-i k_{y a} y\right) \quad(y>0), \\
u_{1}=a_{3}^{(1)} \exp \left[i k_{y s}(y+d)\right] \quad(y<-d),
\end{gathered}
$$

где $k_{y a}=k_{0} n_{a} \cos \theta, k_{y s}=k_{0} \sqrt{\varepsilon_{s}-\varepsilon_{a} \sin ^{2} \theta}, \varepsilon_{s}=\left(n_{s}-i k_{s}\right)^{2}$, $a_{5}^{(1)}, b_{5}^{(1)}, a_{3}^{(1)}-$ некоторые константы. Для конкретизации последних констант заметим, что задача Коши для системы (10) на отрезке $-d \leq y \leq 0$ при начальных условиях $u_{1}(-d)=1, v_{1}(-d)=i k_{y s}$ имеет единственное решение $u=u_{1}(y), v=v_{1}(y)$. Если это решение найдено, то в силу (11), (12) и линейности уравнений (10) можно записать

$$
\begin{gathered}
b_{5}^{(1)}=r_{53}^{(1)} a_{5}^{(1)}, \\
r_{53}^{(1)}=\left[i k_{y a} u_{1}(0)-v_{1}(0)\right]\left[i k_{y a} u_{1}(0)+v_{1}(0)\right]^{-1}, \\
a_{3}^{(1)}=t_{53}^{(1)} a_{5}^{(1)}, \quad t_{53}^{(1)}=2 i k_{y a}\left[i k_{y a} u_{1}(0)+v_{1}(0)\right]^{-1} .
\end{gathered}
$$

Здесь $r_{53}^{(1)}$ и $t_{53}^{(1)}$ имеют смысл амплитудных коэффициентов отражения и прохождения плоской волны $s$-поляризации, падающей из области $y>0$ на слой 4 (рис. 1), 
расположенный на полуограниченной подложке, а $a_{5}^{(1)}$ является амплитудой частного решения.

Для второго частного решения $\left(u=u_{2}(y), v=v_{2}(y)\right)$ имеем

$$
\begin{gathered}
u_{2}=b_{5}^{(2)} \exp \left(-i k_{y a} y\right) \quad(y>0) \\
u_{2}=a_{3}^{(2)} \exp \left[i k_{y s}(y+d)\right]+b_{3}^{(2)} \exp \left[-i k_{y s}(y+d)\right] \\
(-d-D \leq y \leq-d) \\
a_{3}^{(2)}=r_{35}^{(1)} b_{3}^{(2)} \\
r_{35}^{(1)}=\left[i k_{y s} u_{2}(-d)+v_{2}(-d)\right]\left[i k_{y s} u_{2}(-d)-v_{2}(-d)\right]^{-1} \\
b_{5}^{(2)}=t_{35}^{(1)} b_{3}^{(2)} \\
t_{35}^{(1)}=2 i k_{y s}\left[i k_{y s} u_{2}(-d)-v_{2}(-d)\right]^{-1}
\end{gathered}
$$

где $u_{2}(y), v_{2}(y)$ - решение задачи Коши для системы (10) на промежутке $-d \leq y \leq 0$ при начальных условиях $u_{2}(0)=1, v_{2}(0)=-i k_{y a}$. В (17) и (18) $r_{35}^{(1)}$ и $t_{35}^{(1)}$ имеют смысл амплитудных коэффициентов отражения и прохождения плоской волны $s$-поляризации, падающей на слой 4 со стороны подложки, а $b_{3}^{(2)}$ является амплитудой решения.

В окрестности слоя 2, занимающего область $-d^{\prime}-d-D<y<-d-D$ (рис. 1 ), с учетом условия излучения Зоммерфельда при $y \rightarrow-\infty$ имеем

$$
\begin{gathered}
u=A \exp \left[i k_{y s}(y+d+D)\right]+B \exp \left[-i k_{y s}(y+d+D)\right] \\
(-d-D \leq y \leq-d) \\
u=C \exp \left[i k_{y a}\left(y+d+D+d^{\prime}\right)\right] \quad\left(y \leq-d-D-d^{\prime}\right) .
\end{gathered}
$$

Здесь $A-$ некоторая константа,

$$
\begin{gathered}
B=r_{31}^{(1)} A, \\
r_{31}^{(1)}=\left[i k_{y s} u(-d-D)-v(-d-D)\right] \\
\times\left[i k_{y s} u(-d-D)+v(-d-D)\right]^{-1}, \\
C=t_{31}^{(1)} A, \\
t_{31}^{(1)}=2 i k_{y s}\left[i k_{y s} u(-d-D)+v(-d-D)\right]^{-1},
\end{gathered}
$$

$r_{31}^{(1)}$ и $t_{31}^{(1)}$ имеют смысл амплитудных коэффициентов отражения и прохождения плоской волной $s$-поляризации, падающей из полуограниченной области $y>-d-D$, слоя 2 (рис. 1), а под $u(y), v(y)$ понимается решение задачи Коши для системы (10) на промежутке $-d^{\prime}-d-D \leq y \leq-d-D$ при начальных условиях $u\left(-d^{\prime}-D-d\right)=1, v\left(-d^{\prime}-D-d\right)=i k_{y a}$.

Поскольку в области $-d-D \leq y \leq-d$ должно выполняться тождество $u(y) \equiv u_{1}(y)+u_{2}(y)$, из (12), (14), (16), (17), (19), (21) заключаем, что

$$
A=\exp \left(-i k_{y s} D\right)\left(t_{53}^{(1)} a_{5}^{(1)}+r_{35}^{(1)} b_{3}^{(2)}\right),
$$

$$
\begin{aligned}
b_{3}^{(2)}= & a_{5}^{(1)} r_{31}^{(1)} t_{53}^{(1)} \exp \left(-2 i k_{y s} D\right) \\
& \times\left[1-r_{31}^{(1)} r_{35}^{(1)} \exp \left(-2 i k_{y s} D\right)\right]^{-1} .
\end{aligned}
$$

Из (11), (15), (20) очевидно, что $\hat{E}_{i}^{(1)}=a_{5}^{(1)}, \hat{E}_{r}^{(1)}=$ $=b_{5}^{(1)}+b_{5}^{(2)}, \hat{E}_{t}^{(1)}=C$. Поэтому учитывая (13), (18), (23), (24), получаем

$$
\begin{aligned}
r_{\alpha}(\theta, \lambda)= & r_{53}^{(\alpha)}+r_{31}^{(\alpha)} t_{35}^{(\alpha)} t_{53}^{(\alpha)} \exp \left(-2 i k_{y s} D\right) \\
& \times\left[1-r_{31}^{(\alpha)} r_{35}^{(\alpha)} \exp \left(-2 i k_{y s} D\right)\right]^{-1} . \\
t_{\alpha}(\theta, \lambda)= & t_{53}^{(\alpha)} t_{31}^{(\alpha)} \exp \left(-i k_{y s} D\right) \\
& \times\left[1-r_{31}^{(\alpha)} r_{35}^{(\alpha)} \exp \left(-2 i k_{y s} D\right)\right]^{-1},
\end{aligned}
$$

где $\alpha=1$. Ключевое значение для применения формул (25), (26) имеет нахождение входящих в них коэффициентов $r_{53}^{(\alpha)}, r_{35}^{(\alpha)}, r_{31}^{(\alpha)}, t_{35}^{(\alpha)}, t_{53}^{(\alpha)}, t_{31}^{(\alpha)}$ путем интегрирования упомянутых выше задач Коши. При рассмотрении неоднородных слоев 2 и 4 эти задачи могут быть решены либо методом Рунге-Кутта, либо методом стратификации [16]. В последнем случае неоднородный слой заменяется последовательностью $n$ либо $m$ тонких однородных слоев (рис. 1) с диэлектрическими проницаемостями, осуществляющими ступенчатую интерполяцию диэлектрической проницаемости неоднородного слоя. В принципе значения напряженности электрического поля на границах слоев могут быть рассчитаны путем перемножения $2 \times 2$ матриц [8,9], но ниже используется иная схема метода стратификации, основанная на рекуррентных соотношениях, требующая меньшего объема вычислений [16].

Для коэффициентов $r_{53}^{(\alpha)}$ и $t_{53}^{(\alpha)}$ вида $(13),(14)$ имеем $u_{1}(0)=u_{1}^{(n+1)}, v_{1}(0)=v_{1}^{(n+1)}$,

$$
\begin{array}{r}
u_{1}^{(j+1)}=u_{1}^{(j)} \cos \left(k_{y j} d_{j}\right)+v_{1}^{(j)} \sin \left(k_{y j} d_{j}\right), \\
v_{1}^{(j+1)}=-u_{1}^{(j)} k_{y j} \sin \left(k_{y j} d_{j}\right)+v_{1}^{(j)} \cos \left(k_{y j} d_{j}\right),
\end{array}
$$

где $j=1,2, \ldots, n, k_{y j}=k_{0} \sqrt{\varepsilon_{j}-\varepsilon_{\alpha} \sin ^{2} \theta}, \varepsilon_{j}$ и $d_{j}-$ диэлектрическая проницаемость и толщина $j$-го слоя $\left(\sum_{j=1}^{n} d_{j}=d\right), u_{1}^{(1)}=1, v_{1}^{(1)}=i k_{y s}, u_{1}^{(j)}=u_{1}\left(-d+\sum_{l=1}^{j-1} d_{l}\right)$, $v_{1}^{(j)}=v_{1}\left(-d+\sum_{l=1}^{j-1} d_{l}\right)$. В частном случае, когда слой 4 (рис. 1) однороден $(n=1)$, из (27) получаем

$$
\begin{gathered}
u_{1}(0)=\cos \left(k_{y f} d\right)+i k_{y s} k_{y f}^{(-1)} \sin \left(k_{y f} d\right), \\
v_{1}(0)=-k_{y f} \sin \left(k_{y f} d\right)+i k_{y s} \cos \left(k_{y f} d\right),
\end{gathered}
$$

где $k_{y f}=k_{0} \sqrt{\varepsilon_{f}-\varepsilon_{a} \sin ^{2} \theta}, \varepsilon_{f}=\left(n_{f}-i k_{f}\right)^{2}-$ диэлектрическая проницаемость слоя 4 .

При вычислении коэффициентов $r_{35}^{(1)}$ и $t_{35}^{(1)}$ вида (17), (18) имеем $u_{2}(-d)=u_{2}^{(0)}, v_{2}(-d)=v_{2}^{(0)}$,

$$
u_{2}^{j-1}=u_{2}^{(j)} \cos \left(k_{y j} d_{j}\right)-v_{2}^{(j)} k_{y j}^{-1} \sin \left(k_{y j} d_{j}\right),
$$




$$
v_{2}^{j-1}=u_{2}^{(j)} k_{y j} \sin \left(k_{y j} d_{j}\right)+v_{2}^{(j)} \cos \left(k_{y j} d_{j}\right),
$$

где $j=n, n-1, \ldots, 1, \quad u_{2}^{(n)}=1, \quad v_{2}^{(n)}=-i k_{y a}, \quad u_{2}^{(j)}=$ $=u_{2}\left(-d+\sum_{l=1}^{j} d_{l}\right), v_{2}^{(j)}=v_{2}\left(-d+\sum_{l=1}^{j} d_{l}\right)$. При $n=1$ (однородный слой 4)

$$
\begin{aligned}
& u_{2}(-d)=\cos \left(k_{y j} d\right)+i k_{y a} k_{y f}^{(-1)} \sin \left(k_{y f} d\right), \\
& v_{2}(-d)=k_{y f} \sin \left(k_{y f} d\right)-i k_{y a} \cos \left(k_{y f} d\right) .
\end{aligned}
$$

В формулах (21), (22) для коэффициентов слоя 2 величины $u(-d-D)=u^{(m+1)}, v(-d-D)=u^{(m+1)}$ рассчитываются по рекуррентным формулам, аналогичным (27):

$$
\begin{aligned}
& u_{1}^{(j+1)}=u_{1}^{(j)} \cos \left(k_{y j} d_{j}\right)+v_{1}^{(j)} k_{y j}^{(-1)} \sin \left(k_{y j} d_{j}\right), \\
& v_{1}^{(j+1)}=-u_{1}^{(j)} k_{y j} \sin \left(k_{y j} d_{j}\right)+v_{1}^{(j)} \cos \left(k_{y j} d_{j}\right),
\end{aligned}
$$

где $j=1,2, \ldots, m, \sum_{j=1}^{m} d_{j}=d^{\prime}, \quad u_{1}^{(1)}=1, \quad v_{1}^{(1)}=i k_{y a}$, $u_{1}^{(j)}=u_{1}\left(-d-D-d^{\prime}+\sum_{l=1}^{j-1} d_{l}\right), v_{1}^{(j)}=v_{1}\left(-d-D-d^{\prime}+\right.$ $\left.+\sum_{l=1}^{j-1} d_{l}\right)$

Если слой 2 однородный $(m=1)$, то

$$
\begin{gathered}
u(-d-D)=\cos \left(k_{y f}^{\prime} d^{\prime}\right)+i k_{y a} k_{y f}^{\prime-1} \sin \left(k_{y f}^{\prime} d^{\prime}\right), \\
v(-d-D)=-k_{y f}^{\prime} \sin \left(k_{y f}^{\prime} d^{\prime}\right)+i k_{y a} \cos \left(k_{y f}^{\prime} d^{\prime}\right),
\end{gathered}
$$

где $k_{y f}^{\prime}=k_{0} \sqrt{\varepsilon_{f}^{\prime}-\varepsilon_{a} \sin ^{2} \theta}, \varepsilon_{f}^{\prime}$ - диэлектрическая проницаемость слоя 2.

Рассмотрение волн $p$-поляризации может быть проведено по аналогичной схеме. В этом случае формулы (25), (26), где $\alpha=2$, сохраняют свой вид. Однако при расчете коэффициентов в (25), (26) вместо (10) должна интегрироваться система [16]

$$
\begin{gathered}
d u / d y=\varepsilon(y) v, \\
d v / d y=-k_{0}^{2}[\varepsilon(y)]^{-1}\left[\varepsilon(y)-\varepsilon_{a} \sin ^{2} \theta\right] u,
\end{gathered}
$$

где $u$ имеет смысл $x$-компоненты магнитного поля. Для коэффициентов отражения и прохождения волн $p$-поляризации имеем

$$
\begin{gathered}
r_{53}^{(2)}=\left[i k_{y a} u_{1}(0)-\varepsilon_{a} v_{1}(0)\right]\left[i k_{y a} u_{1}(0)+\varepsilon_{a} v_{1}(0)\right]^{-1}, \\
t_{53}^{(2)}=2 i k_{y a}\left[i k_{y a} u_{1}(0)+\varepsilon_{a} v_{1}(0)\right]^{-1}, \\
r_{35}^{(2)}=\left[i k_{y s} u_{2}(-d)+\varepsilon_{s} v_{2}(-d)\right]\left[i k_{y s} u_{2}(-d)-\varepsilon_{s} v_{2}(-d)\right]^{-1}, \\
t_{35}^{(2)}=2 i k_{y s}\left[i k_{y s} u_{2}(-d)-\varepsilon_{s} v_{2}(-d)\right]^{-1}, \\
r_{31}^{(2)}=\left[i k_{y s} u(-d-D)-\varepsilon_{s} v(-d-D)\right] \\
\times\left[i k_{y s} u(-d-D)+\varepsilon_{s} v(-d-D)\right]^{-1}
\end{gathered}
$$

$$
t_{31}^{(2)}=2 i k_{y s}\left[i k_{y s} u(-d-D)+\varepsilon_{s} v(-d-D)\right]^{-1} .
$$

Здесь $u_{1}(0)=u_{1}^{(n+1)}, v_{1}(0)=v_{1}^{(n+1)}$,

$$
\begin{gathered}
u_{1}^{(j+1)}=u_{1}^{(j)} \cos \left(k_{y j} d_{j}\right)+v_{1}^{(j)} \varepsilon_{j} k_{y j}^{-1} \sin \left(k_{y j} d_{j}\right), \\
v_{1}^{(j+1)}=-u_{1}^{(j)} \varepsilon_{j}^{-1} k_{y j} \sin \left(k_{y j} d_{j}\right)+v_{1}^{(j)} \cos \left(k_{y j} d_{j}\right),
\end{gathered}
$$

где $j=1,2, \ldots, n, u_{1}^{(1)}=1, v_{1}^{(1)}=i k_{y s} \varepsilon_{s}^{-1}$, случаю $n=1$ соответствует

$$
\begin{gathered}
u_{1}(0)=\cos \left(k_{y f} d\right)+i k_{y s} \varepsilon_{s}^{-1} \varepsilon_{f} k_{y f}^{-1} \sin \left(k_{y f} d\right), \\
v_{1}(0)=-k_{y f} \varepsilon_{f}^{-1} \sin \left(k_{y f} d\right)+i k_{y s} \varepsilon_{s}^{-1} \cos \left(k_{y f} d\right) ; \\
u_{2}(-d)=u_{2}^{(0)}, \quad v_{2}(-d)=v_{2}^{(0)}, \\
u_{2}^{j-1}=u_{2}^{(j)} \cos \left(k_{y j} d_{j}\right)-v_{2}^{(j)} \varepsilon_{j} k_{y j}^{-1} \sin \left(k_{y j} d_{j}\right), \\
v_{2}^{(j-1)}=u_{2}^{(j)} k_{y j} \varepsilon_{j}^{-1} \sin \left(k_{y j} d_{j}\right)+v_{2}^{(j)} \cos \left(k_{y j} d_{j}\right) ;
\end{gathered}
$$

где $j=n, n-1, \ldots, 1, u_{2}^{(n)}=1, v_{2}^{(n)}=-i k_{y a} \varepsilon_{a}^{-1}$, а при $n=1$

$$
\begin{gathered}
u_{2}(-d)=\cos \left(k_{y f} d\right)+i k_{y a} \varepsilon_{a}^{-1} k_{y f}^{-1} \varepsilon_{f} \sin \left(k_{y f} d\right), \\
v_{2}(-d)=k_{y f} \varepsilon_{f}^{-1} \sin \left(k_{y f} d\right)-i k_{y a} \varepsilon_{a}^{-1} \cos \left(k_{y f} d\right) ; \\
u(-d-D)=u^{(m+1)}, \quad v(-d-D)=u^{(m+1)}, \\
u^{(j+1)}=u^{(j)} \cos \left(k_{y j} d_{j}\right)+v^{(j)} \varepsilon_{j} k_{y j}^{-1} \sin \left(k_{y j} d\right), \\
v_{1}^{(j+1)}=-u_{1}^{(j)} k_{y j} \varepsilon_{j}^{-1} \sin \left(k_{y j} d_{j}\right)+v_{1}^{(j)} \cos \left(k_{y j} d_{j}\right) ;
\end{gathered}
$$

где $j=1,2, \ldots, m, u^{(1)}=1, v^{(1)}=i k_{y a} \varepsilon_{a}^{-1}$, а при $m=1$.

$$
\begin{gathered}
u(-d-D)=\cos \left(k_{y f}^{\prime} d^{\prime}\right)+\frac{i k_{y a} \varepsilon_{f}^{\prime}}{\varepsilon_{a} k_{y f}^{\prime}} \sin \left(k_{y f}^{\prime} d^{\prime}\right), \\
v(-d-D)=-\frac{k_{y f}^{\prime}}{\varepsilon_{f}^{\prime}} \sin \left(k_{y f}^{\prime} d^{\prime}\right)+i \frac{k_{y a}}{\varepsilon_{a}} \cos \left(k_{y f}^{\prime} d^{\prime}\right) .
\end{gathered}
$$

Рассмотрим возможности упрощения выражений (8) и (9). В большинстве практических ситуаций толщины слоев $d$ и $d^{\prime}$ сопоставимы с $\lambda$, а $D \gg h$. Тогда при выполнении условия (1) коэффициенты отражения и прохождения слоев 2 и 4 в правых частях (25) и (26) пренебрежимо мало изменяются на эффективном промежутке интегрирования $\omega_{0}-\Delta \omega \leq \omega_{0}+\Delta \omega$ в (8) и (9). При естественном условии $2 n_{s} k_{s} \ll \chi^{2}$, где $\chi=\sqrt{n_{s}^{2}-\varepsilon_{a} \sin ^{2} \theta}$, экспоненциальный член в (26) представим в виде

$$
\exp \left(-i k_{y s} D\right)=\exp \left(-n_{s} k_{s} \chi^{-1} k_{0} D\right) \exp \left(-i \chi k_{0} D\right),
$$

где в случае слабо поглощающей подложки $n_{s} k_{s} \chi^{-1} k_{0} D \leq 1$. Тогда относительное изменение функции $\exp \left(-n_{s} k_{s} \chi^{-1} k_{0} D\right)$ на эффективных промежутках интегрирования в числителях правых частей (8) и (9), равное $n_{s} k_{s} \chi^{-1} k_{0} D \Delta \omega \omega_{0}^{-1}$, будет пренебрежимо малым. $\mathrm{B}$ такой ситуации необходимо оценить влияние на 
величины интегралов в (8) и (9) быстро осциллирующих экспонент $\exp \left(-i \chi k_{0} D\right)$ и $\exp \left(-2 i \chi k_{0} D\right)$. Для этого заметим, что в силу закона сохранения энергии выполняется неравенство $\left|r_{31}^{(\alpha)} r_{35}^{(\alpha)} \exp \left(-2 i k_{y s} D\right)\right|<1$. В результате можно записать ряд геометрической прогрессии

$$
\begin{aligned}
& {\left[1-r_{31}^{(\alpha)} r_{35}^{(\alpha)} \mid \exp \left(-2 i k_{y s} D\right)\right]^{-1}} \\
& =\sum_{\kappa=0}^{\infty}\left[r_{31}^{(\alpha)} r_{35}^{(\alpha)} \mid \exp \left(-2 i k_{y s} D\right)\right]^{\kappa}
\end{aligned}
$$

Подстановка (29) в (25) дает

$$
\begin{aligned}
& \left|r_{\alpha}(\theta, \lambda)\right|^{2}=\left\{\left|r_{53}^{(\alpha)}\right|^{2}+\left|r_{31}^{(\alpha)} S_{\alpha} \exp \left(-2 i k_{y}^{(s)} D\right)\right|^{2}\right. \\
& \left.+r_{53}^{(\alpha) *} r_{31}^{(\alpha)} S_{\alpha} \exp \left(-2 i k_{y}^{(s)} D\right)+r_{53}^{(\alpha)} r_{31}^{(\alpha) *} S_{\alpha}^{*} \exp \left(2 i k_{y}^{(s)} D\right)\right\} \\
& \times \sum_{l=0}^{\infty} \sum_{j=0}^{\infty}\left(r_{31}^{(\alpha)} r_{35}^{(\alpha)}\right)^{l}\left(r_{31}^{(\alpha) *} r_{35}^{(\alpha) *}\right)^{j} \exp \left[2 i D\left(j k_{y s}^{*}-l k_{y s}\right)\right],
\end{aligned}
$$

где $S_{\alpha}=t_{35}^{(\alpha)} t_{53}^{(\alpha)}-r_{35}^{(\alpha)} r_{53}^{(\alpha)}$. Из (30) следует, что подынтегральная функция в числителе правой части (8) представляется суммой практически постоянных на эффективном промежутке интегрирования и быстро осциллирующих слагаемых. Интегралы, содержащие слагаемые второго типа после вынесения из-под них слабо изменяющихся множителей, оказываются пропорциональными интегралам вида

$$
I_{\alpha}\left(\eta_{l}\right)=\int_{-\infty}^{\infty} G_{\alpha}(x) \exp \left(i \eta_{l} x\right) d x,
$$

где $\eta_{l}=l 4 \pi \chi D \Delta \lambda \lambda_{0}^{-2}, l= \pm 1, \pm 2, \ldots$. Согласно методу стационарной фазы [17], $I_{\alpha}\left(\eta_{l}\right) \rightarrow 0$ при $\eta_{1} \gg 1$ или

$$
D \gg \lambda_{0}^{2}(4 \pi \chi \Delta \lambda)^{-1} \text {. }
$$

Таким образом, при условии (31) для оценки $R_{\alpha}\left(\theta, \lambda_{0}\right)$ достаточно учесть только слабо изменяющиеся на эффективном промежутке интегрирования слагаемые в (30). Очевидно, что такие слагаемые образуются из членов двойной суммы в (30), у которых и $j=l \pm 1$. Они формируют три бесконечных геометрических прогрессии. После вынесения из-под интеграла в числителе правой части (8) сумм этих прогрессий получаем аналитическое приближение

$$
\begin{aligned}
& R_{\alpha}(\theta, \lambda)=\left|r_{53}^{(\alpha)}\right|^{2}+\left|r_{31}^{(\alpha)} t_{35}^{(\alpha)} t_{53}^{(\alpha)} \exp \left(-2 i k_{y}^{(s)} D\right)\right|^{2} \\
& \times\left[1-\left|r_{31}^{(\alpha)} r_{35}^{(\alpha)} \exp \left(-2 i k_{y}^{(s)} D\right)\right|^{2}\right]^{-1} .
\end{aligned}
$$

Аналогичное приближение для (9) имеет вид

$$
\begin{aligned}
T_{\alpha}(\theta, \lambda)= & \left|t_{53}^{(\alpha)} t_{31}^{(\alpha)} \exp \left(-i k_{y s} D\right)\right|^{2} \\
& \times\left[1-\left|r_{31}^{(\alpha)} r_{35}^{(\alpha)} \exp \left(-2 i k_{y s} D\right)\right|^{2}\right]^{-1}
\end{aligned}
$$

При $\theta=0$ индекс $\alpha$ в (32), (33) становится несущественным. В этом случае данные формулы могут быть сопоставлены с результатами работ $[8,11]$. Сравнение показало, что они находятся в полном согласии с аналогичными формулами, полученными суммированием рядов Эйри в [8], а их численное тестирование для различных структур однородная пленка-подложка (случай $\left.n=1, d^{\prime}=0\right)$ привело к результатам, совпадающим в 16 десятичных разрядах с результатами расчетов по формулам работы [11].

Отметим еще две возможности упрощения (8) и (9). Во-первых, если в $(28) \exp \left(-n_{s} k_{s} \chi^{-1} k_{0} D\right) \rightarrow 0$ (случай непрозрачной подложки), то согласно (8), (9), (25), (26),

$$
R_{\alpha}\left(\theta, \lambda_{0}\right)=\left|r_{53}^{(\alpha)}\right|^{2}, \quad T_{\alpha}\left(\theta, \lambda_{0}\right) \rightarrow 0,
$$

т. е. измеримыми являются только спектры когерентной отражательной способности слоя 4. Во-вторых, из (25), (28) следует, что при условии $D \ll \lambda_{0}^{2}(\chi \Delta \lambda)^{-1}$ функция $\left|r_{\alpha}(\theta, \lambda)\right|^{2}$ пренебрежимо мало изменяется на эффективном промежутке интегрирования в (8) и может быть вынесена из-под интеграла. Такой же результат получается и для функции $\left|t_{\alpha}(\theta, \lambda)\right|^{2}$ в (9). Следовательно, если толщина подложки сопоставима с длиной волны, то может быть использовано монохроматическое приближение

$$
R_{\alpha}(\theta, \lambda)=\left|r_{\alpha}(\theta, \lambda)\right|^{2}, \quad T_{\alpha}(\theta, \lambda)=\left|t_{\alpha}(\theta, \lambda)\right|^{2} .
$$

Приближения (32)-(35) не требуют знания аппаратной функции спектрофотометра и удобны для решения обратных задач спектрофотометрии слоев. Но их применимость в конкретных ситуациях должна проверяться прямым расчетом спектров (8) и (9). В частности, оценка (31) существенно зависит от гладкости функции $G_{\alpha}(x)[16]$. Это подтверждается выражениями $I_{\alpha}\left(\eta_{l}\right)=\sqrt{\pi} \exp \left(-0.25 \eta_{l}^{2}\right), I_{\alpha}\left(\eta_{l}\right)=2 \eta_{l}^{-1} \sin \eta_{l}$, относящимися к распространенным гауссовой,

$$
G_{\alpha}(x)=\exp \left(-x^{2}\right)
$$

и прямоугольной,

$$
G_{\alpha}(x)=1 \quad \text { при } \quad|x| \leq 1, \quad G_{\alpha}(x) \equiv 0 \quad \text { при } \quad|x| \geq 1,
$$

моделям аппаратной функции спектрофотометра соответственно.

Примеры использования полученных соотношений и оценки их эффективности представлены в следующем разделе.

\section{Решение обратной задачи спектрофотометрии для слоя $\mathrm{ZnO}: \mathrm{Al}$}

Ниже исследован слой $\mathrm{ZnO}: \mathrm{Al}$ с 2\% содержанием алюминия, нанесенный магнетронным распылением мишени $\mathrm{ZnO}: \mathrm{Al}_{2} \mathrm{O}_{3}$ на плоскопараллельную подложку 
из технического стекла толщиной $D=2.14 \mathrm{~mm}$. Ориентировочно толщина слоя находилась в диапазоне $300 \mathrm{~nm}<d<400 \mathrm{~nm}$. Детали технологии описаны в [19].

Измерения спектров

$$
R_{1}(\theta, \lambda), \quad R_{2}(\theta, \lambda), \quad T_{1}(\theta, \lambda), \quad T_{2}(\theta, \lambda)
$$

чистой подложки и подложки с названным слоем выполнены при двух углах падения $\theta=\theta_{1}=8^{\circ}$ и $\theta=\theta_{2}=40^{\circ}$ на спектрофотометре Photon RT („ЭссентОптикс“, Беларусь) при $\Delta \lambda=2 \mathrm{~nm}$ и относительной среднеквадратичной ошибке для отражательной и пропускательной способностей $q=0.0043$. Исследован диапазон $\lambda_{\min } \leq \lambda \leq \lambda_{\max }$, где $\lambda_{\min }=380 \mathrm{~nm}, \lambda_{\max }=950 \mathrm{~nm}$. Спектры (38) были измерены с шагом $2 \mathrm{~nm}$ при одинаковых длинах волн в количестве $M=286$.

Перед определением характеристик слоя были найдены спектры показателей преломления и поглощения подложки $n_{s}(\lambda)$ и $k_{s}(\lambda)$. Возможность нахождения $k_{s}(\lambda)$ по функциям $R(\lambda)$ и $T(\lambda)$ была указана в [1]. Но схема, предложенная в [1], имеет ограниченную область применимости, поскольку относится к случаю нормального падения излучения на структуру и предполагает использование априори известного распределения $n_{s}(\lambda)$. Для формулировки более общего алгоритма, который необходим, если $\theta \neq 0$, а функция $n_{s}(\lambda)$ подлежит определению, заметим, что выражения (32), (33) при $\alpha=1$, $n=1, m=1, d=0, d^{\prime}=0$ могут быть приведены к виду

$$
\begin{aligned}
R_{1}(\theta, \lambda)= & \left|r_{53}^{(1)}\right|^{2}\left\{1+\left[1-2 \operatorname{Re}\left(r_{53}^{(1)}\right)^{2}\right]\left|\exp \left(-i k_{y s} D\right)\right|^{4}\right\} \\
& \times\left[1-\left|r_{53}^{(1)} \exp \left(-i k_{y s} D\right)\right|^{4}\right]^{-1},
\end{aligned}
$$$$
T_{1}(\theta, \lambda)=\left|\exp \left(-i k_{y s} D\right)^{2}\right|\left[1+\left|r_{53}^{(1)}\right|-2 \operatorname{Re}\left(r_{53}^{(1)}\right)^{2}\right]
$$$$
\times\left[1-\left.\left|r_{53}^{(1)}\right| \exp \left(-i k_{y s} D\right)\right|^{4}\right]^{-1},
$$

где $r_{53}^{(1)}=\left(k_{y a}-k_{y s}\right)\left(k_{y s}+k_{y s}\right)^{-1}$. Предполагая, что $k_{s} \ll 1$, из (39), (40) получаем аналитическое решение обратной задачи об определении спектров $n_{s}(\lambda)$ и $k_{s}(\lambda)$ при известном значении $D$ :

$$
\begin{aligned}
n_{s}= & n_{a} \sqrt{\sin ^{2} \theta+\left[(1+\sqrt{\rho})(1-\sqrt{\rho})^{-1} \cos \theta\right]^{2}} \\
& \times\left[1+O\left(k_{s}^{2}\right)\right], \\
k_{s}=- & \chi\left(2 n_{s} k_{0} D\right)^{-1} \ln \left\{\left[R_{1}(\theta, \lambda)-\rho\right]\left[T_{1}(\theta, \lambda) \rho\right]^{-1}\right\} \\
& \times\left[1+O\left(k_{s}^{2}\right)\right], \\
\rho=- & 0.5 F-\sqrt{0.25 F^{2}-R_{1}(\theta, \lambda)\left[2-R_{1}(\theta, \lambda)\right]^{-1}}, \\
F= & \left\{\left[R_{1}(\theta, \lambda)-T_{1}(\theta, \lambda)-1\right]\right. \\
& {\left.\left[R_{1}(\theta, \lambda)+T_{1}(\theta, \lambda)-1\right]-2\right\}\left[2-R_{1}(\theta, \lambda)\right]^{-1} . }
\end{aligned}
$$

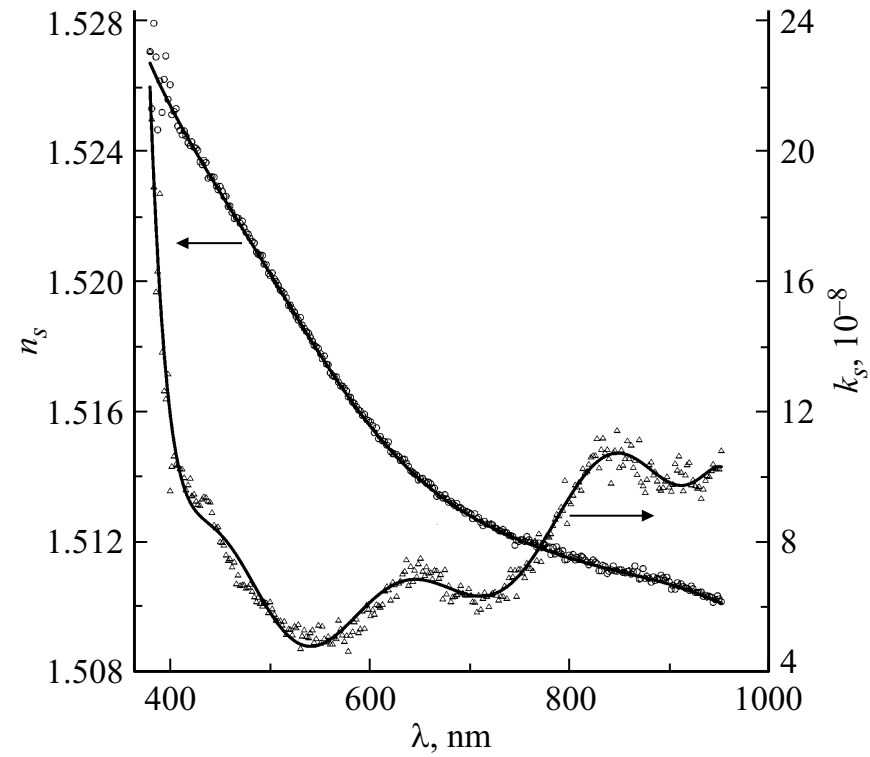

Рис. 2. Восстановленные спектры показателей преломления и поглощения стеклянной плоскопараллельной подложки.

Результаты обработки эксперимента при $\theta=8^{\circ}$, $\theta=40^{\circ}, n_{a}=1.0003$ на основании формул (41), (42) после пренебрежения в них малыми $O\left(k_{s}^{2}\right)$ представлены на рис. 2. Здесь дискретные значки (кружки для $n_{s}$, треугольники для $k_{s}$ ) рассчитаны в результате подстановки в (41), (42) экспериментальных данных для $R_{1}(\theta, \lambda), T_{1}(\theta, \lambda)$; сплошные кривые - интерполяция полученных дискретных зависимостей полиномами 9-й степени. В дальнейших расчетах функции $n_{s}(\lambda)$ и $k_{s}(\lambda)$ представлялись этими полиномами и считались заданными.

При определении параметров слоя $\mathrm{ZnO}: \mathrm{Al}$ использовалась модель однородного слоя на подложке $(n=1$, $d^{\prime}=0 \mathrm{~nm}$ ). Комплексный показатель преломления слоя представлялся многочленом Лагранжа-Чебышева степени $L$ без привлечения каких-либо физических моделей дисперсии материала:

$$
\begin{gathered}
n_{f}(\lambda)-i k_{f}(\lambda)=\sum_{j=0}^{L}\left[n_{f}\left(\lambda_{j}\right)-i k_{f}\left(\lambda_{j}\right)\right] P_{j}(\lambda), \\
P_{j}(\lambda)=\left[\prod_{l=0, l \neq j}^{L}\left(\lambda-\lambda_{l}\right)\right]\left[\prod_{l=0, l \neq j}^{L}\left(\lambda_{j}-\lambda_{l}\right)\right]^{-1}, \\
\lambda_{j}=0.5\left(\lambda_{\max }-\lambda_{\min }\right) \cos \left[0.5 \pi(2 L-2 j+1)(L+1)^{-1}\right]+ \\
\quad+0.5\left(\lambda_{\max }+\lambda_{\min }\right) .
\end{gathered}
$$

Таким образом, модель слоя содержала $2 L+3$ свободных параметра $X_{k}: X_{1}=d, X_{k}=n_{f}\left(\lambda_{j}\right)$ при $k=i+2$, $X_{k}=k_{f}\left(\lambda_{j}\right)$ при $k=i+L+3$, где $i=0,1, \ldots, L$. Эти параметры определялись методом наименьших квадра- 
тов с целевой функцией

$$
\Phi\left(X_{1}, \ldots, X_{2 L+3}\right)=\sum_{\alpha=1}^{2} \sum_{\beta=1}^{2} \sum_{\delta=1}^{2} \sum_{j=1}^{M}\left(\Omega_{\alpha \beta \delta j}^{(e)}-\Omega_{\alpha \beta \delta j}\right)^{2},
$$

где $\Omega_{\alpha \beta 1 j}=R_{\alpha}\left(\theta_{\beta}, \lambda_{j}\right), \Omega_{\alpha \beta 2 j}=T_{\alpha}\left(\theta_{\beta}, \lambda_{j}\right)$ - отражательная и пропускательная способности структуры, рассчитываемые на основании модели (32), (33); $\Omega_{\alpha \beta \delta j}^{(e)}-$ соответствующие экспериментальные данные. Использование в (44) отражательных и пропускательных способностей структуры при двух углах падения и двух состояниях поляризации света позволяет существенно снизить вероятность появления ложных решений при решении обратной оптической задачи [18].

В нулевом приближении перебором значений аргументов с грубым шагом определялся глобальный минимум функции (44) в области $300 \mathrm{~nm}<X_{1}<400 \mathrm{~nm}$, $1.7 \leq X_{2, \ldots, L+2} \leq 2.3, X_{L+3, \ldots, 2 L+3}=0$. Затем полученное решение уточнялось путем минимизации (44) градиентным методом [20]. Такой подход привел к физически корректным решениям обратной задачи при всех $L$ из рассмотренного диапазона $0 \leq L \leq 19$. Однако количественно данные решения различались. Аналогичная картина возникает при интерполяции функций полиномами при наличии ошибок задания значений функций [21]. В такой ситуации необходимо выбрать оптимальное значение $L$ [21]. Для его нахождения был применен критерий, основанный на оценке случайных ошибок определения функции $n_{f}(\lambda)$, который подобен критерию, предложенному в [16] при исследовании профилей пространственной неоднородности слоев.

Для ошибок $\Delta X_{k}$ определения параметров $X_{k}$, вызванных ошибками $\Delta \Omega_{\alpha \beta \delta j}^{(e)}$ измерения значений $\Omega_{\alpha \beta \delta j}^{(e)}$, в линейном приближении можно записать [22]

$$
\Delta X_{k}=\sum_{\alpha=1}^{2} \sum_{\beta=1}^{2} \sum_{\delta=1}^{2} \sum_{j=1}^{M} \Delta \Omega_{\alpha \beta \delta j}^{(e)} \sum_{l=1}^{2 L+3} M_{k l}^{-1} \frac{\partial \Omega_{\alpha \beta \delta j}}{\partial X_{l}},
$$

где $M_{k l}^{-1}$ - матрица, обратная матрице

$$
\begin{aligned}
M_{l k}= & \sum_{\alpha=1}^{2} \sum_{\beta=1}^{2} \sum_{\delta=1}^{2} \sum_{j=1}^{M}\left[\frac{\partial \Omega_{\alpha \beta \delta j}}{\partial X_{l}} \frac{\partial \Omega_{\alpha \beta \delta j}}{\partial X_{k}}\right. \\
& \left.-\frac{\partial^{2} \Omega_{\alpha \beta \delta j}}{\partial X_{l} \partial X_{k}}\left(\Omega_{\alpha \beta \delta j}^{(e)}-\Omega_{\alpha \beta \delta j}\right)\right] .
\end{aligned}
$$

Предполагая, что ошибки $\Delta \Omega_{\alpha \beta \delta j}^{(e)}$ статистически независимы и что среднеквадратичное отклонение $\sigma\left(\Omega_{\alpha \beta \delta j}^{(e)}\right)=$ $=q \Omega_{\alpha \beta \delta j}^{(e)}$, из (43), (45) заключаем, что среднеквадратичная ошибка определения функции $n_{f}(\lambda)$ равна

$$
\begin{aligned}
& \sigma\left[n_{f}(\lambda)\right]=q \\
& \times \sqrt{\sum_{\alpha=1}^{2} \sum_{\beta=1}^{2} \sum_{\delta=1}^{2} \sum_{j=1}^{M}\left[\Omega_{\alpha \beta \delta j} \sum_{l=2}^{L+2} P_{l-2}(\lambda) \sum_{k=1}^{2 L+3} M_{l k}^{-1} \frac{\partial \Omega_{\alpha \beta \delta j}}{\partial X_{k}}\right]^{2}} .
\end{aligned}
$$

Аналогично среднеквадратичная ошибка определения толщины слоя

$$
\sigma(d)=q \sqrt{\sum_{\alpha=1}^{2} \sum_{\beta=1}^{2} \sum_{\delta=1}^{2} \sum_{j=1}^{M}\left(\Omega_{\alpha \beta \delta j} \sum_{k=2}^{2 L+3} M_{1 k}^{-1} \frac{\partial \Omega_{\alpha \beta \delta j}}{\partial X_{k}}\right)^{2}} .
$$

Определим средние по диапазону длин волн среднеквадратичное отклонение показателя преломления слоя при данном $L$ и модуль разности показателей преломления слоя, определенных при соседних значениях $L$ :

$$
\begin{gathered}
\bar{\sigma}=\left(\lambda_{\max }-\lambda_{\min }\right)^{-1} \int_{\lambda_{\min }}^{\lambda_{\max }} \sigma\left[n_{f}(\lambda)\right] d \lambda, \\
\overline{\delta n_{f}}=\left(\lambda_{\max }-\lambda_{\min }\right)^{-1} \int_{\lambda_{\min }}^{\lambda_{\max }}\left|n_{f}(\lambda)\right|_{L}-\left.n_{f}(\lambda)\right|_{L-1} \mid d \lambda .
\end{gathered}
$$

Как известно, $\bar{\sigma}$ возрастает при увеличении $L$, а для $\overline{\delta n_{f}}$ наблюдается противоположная тенденция [16]. В результате в процессе увеличения $L$ при некотором значении $L$ будет выполнено неравенство $\overline{\delta n_{f}}<\bar{\sigma}$, которое означает, что различие между спектрами $n_{f}(\lambda)$, определенными при порядках полинома Лагранжа-Чебышева $L$ и $L-1$, находится в пределах естественного статистического разброса. В таком случае в качестве оптимального порядка полинома следует принять указанное значение $L[16]$.

Описанный критерий иллюстрирует табл. 1, где $\Phi_{\min }-$ минимальные значения целевой функции (44), рассчитанные по описанному алгоритму. Согласно табл. 1, оптимальная степень полинома $L=12$.

Зависимости $n_{f}(\lambda), k_{f}(\lambda)$ вида (43), соответствующие $L=12$, представлены на рис. 3 кривыми 1 и 2. Данные зависимости и значение $d=355.29 \mathrm{~nm}$ (табл. 1) использованы для оценки границ применимости приближений (32), (33) и (35). С этой целью был выполнен

Таблица 1. Восстановление параметров слоя $\mathrm{ZnO}: \mathrm{Al}$

\begin{tabular}{c|l|c|c|c}
\hline$L$ & $\Phi_{\min }$ & $\overline{\delta n_{f}} \cdot 10^{4}$ & $\bar{\sigma} \cdot 10^{4}$ & $d \pm \sigma(d), \mathrm{nm}$ \\
\hline 3 & 0.315 & 18985 & 2.83 & $356.42 \pm 0.05$ \\
4 & 0.172 & 119 & 3.28 & $354.82 \pm 0.05$ \\
5 & 0.100 & 87.7 & 3.45 & $354.70 \pm 0.05$ \\
6 & 0.0490 & 71.8 & 3.58 & $356.55 \pm 0.04$ \\
7 & 0.0250 & 57.3 & 4.04 & $356.61 \pm 0.05$ \\
8 & 0.0148 & 44.9 & 4.10 & $355.73 \pm 0.05$ \\
9 & 0.00828 & 20.7 & 4.38 & $355.43 \pm 0.05$ \\
10 & 0.00523 & 15.3 & 4.55 & $355.30 \pm 0.05$ \\
11 & 0.00463 & 7.96 & 4.68 & $355.22 \pm 0.05$ \\
12 & 0.00453 & 4.53 & 4.85 & $355.29 \pm 0.06$ \\
13 & 0.00445 & 3.71 & 4.93 & $355.34 \pm 0.06$ \\
14 & 0.00440 & 2.72 & 5.12 & $355.32 \pm 0.06$ \\
15 & 0.00436 & 3.24 & 5.27 & $355.32 \pm 0.06$ \\
16 & 0.00435 & 2.69 & 5.40 & $355.30 \pm 0.06$
\end{tabular}


Таблица 2. Оценка применимости приближений (32), (33) и (35) при спектрофотометрии слоя ZnO: $\mathrm{Al}$ на подложках различной толщины

\begin{tabular}{|c|c|c|c|c|c|c|c|c|}
\hline \multirow[b]{3}{*}{$D, \mu \mathrm{m}$} & \multicolumn{4}{|c|}{ Аппаратная функция (36) } & \multicolumn{4}{|c|}{ Аппаратная функция (37) } \\
\hline & \multicolumn{2}{|c|}{$\begin{array}{c}\text { Приближение } \\
\text { (32), (33) }\end{array}$} & \multicolumn{2}{|c|}{$\begin{array}{c}\text { Приближение } \\
\text { (35) }\end{array}$} & \multicolumn{2}{|c|}{$\begin{array}{c}\text { Приближение } \\
\text { (32), (33) }\end{array}$} & \multicolumn{2}{|c|}{$\begin{array}{c}\text { Приближение } \\
\text { (35) }\end{array}$} \\
\hline & $\Phi_{\min }$ & $d, \mathrm{~nm}$ & $\Phi_{\min }$ & $d, \mathrm{~nm}$ & $\Phi_{\min }$ & $d, \mathrm{~nm}$ & $\Phi_{\min }$ & $d, \mathrm{~nm}$ \\
\hline 1 & 13.65 & 357.54 & 0.0024 & 355.50 & 14.51 & 367.50 & 0.0011 & 355.43 \\
\hline 5 & 13.80 & 355.29 & 0.246 & 357.48 & 14.51 & 355.28 & 0.135 & 356.85 \\
\hline 10 & 9.96 & 354.36 & 1.568 & 361.53 & 11.11 & 354.32 & 1.154 & 360.21 \\
\hline 50 & 0.337 & 355.34 & 10.02 & 381.42 & 0.586 & 355.17 & 9.372 & 383.72 \\
\hline 100 & 0.00034 & 355.35 & 12.48 & 372.20 & 0.0565 & 355.37 & 12.67 & 363.48 \\
\hline 1000 & 0.000021 & 355.36 & 11.68 & 399.00 & 0.0018 & 355.33 & 11.68 & 399.00 \\
\hline 2140 & 0.000021 & 355.36 & 12.88 & 363.69 & 0.000042 & 355.34 & 12.87 & 363.68 \\
\hline
\end{tabular}

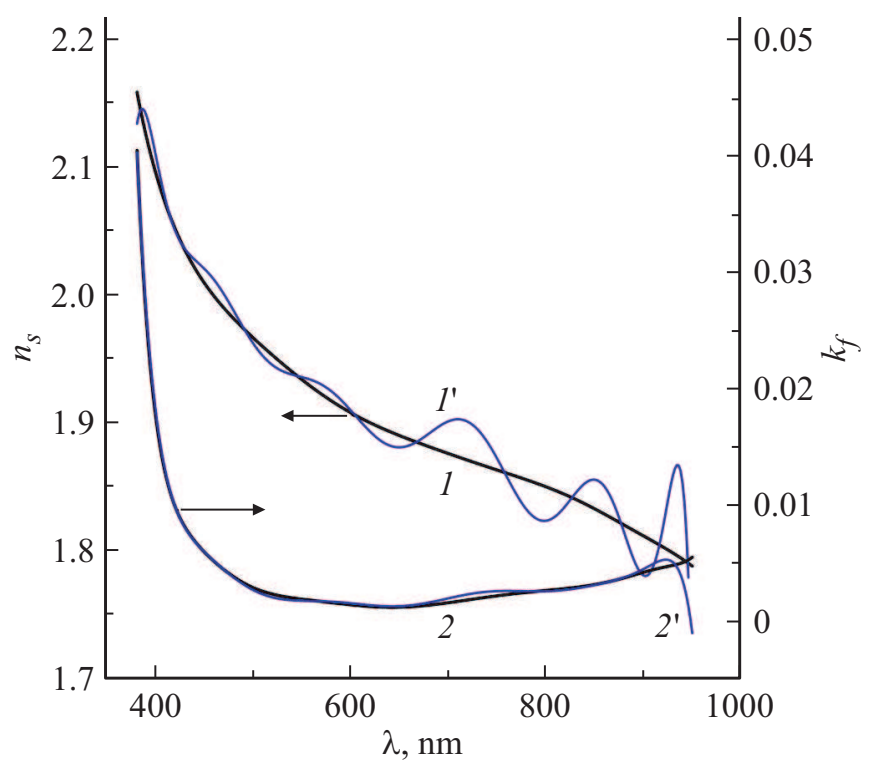

Рис. 3. Восстановленные функции $n_{f}(\lambda)\left(1,1^{\prime}\right)$ и $\left(2,2^{\prime}\right)$ для слоя $\mathrm{ZnO}: \mathrm{Al}$.

вычислительный эксперимент, в котором в качестве „экспериментальных“ спектров $\Omega_{\alpha \beta \delta j}^{(e)}$ принимались результаты их расчета по интегральным формулам (8), (9), при найденных выше параметрах слоя и подложки. Использованы аппаратные функции (36), (37) и указанные ранее значения $\Delta \lambda$ и углов падения. Вычисления выполнены при различных $D$. Решения обратных задач спектрофотометрии слоя на подложках различной толщины найдены при $L=12$ по описанной выше схеме.

На рис. 4 сопоставлены спектры $T_{2}(\theta, \lambda)$ при угле падения $\theta=40^{\circ}$, полученные в вычислительных и реальном экспериментах, а также в результате решения обратных задач спектрофотометрии.

Кривые 1 и 2 на рис. 4, $a$ визуально совпадают и находятся в близком соответствии с экспериментальными данными, однако они имеют мало общего с кривой 3 , рассчитанной в монохроматическом приближении (35). При уменышении толщины подложки до $50 \mu \mathrm{m}$ (рис. $4, b)$ на интегральной кривой 1 возникают колебания, которые в приближении (33) (кривая 2) отсутствуют. Эти колебания, однако, практически не сказываются на восстановлении параметров слоя (см. ниже). В то же время монохроматическое приближение (35) (кривая 3) по-прежнему остается неэффективным. При $D=1 \mu \mathrm{m}$ (рис. 4,c) ситуация качественно изменяется. В данном случае приближение (33) теряет применимость, а корректное восстановление параметров слоя получается в приближении (35).

Интегральную оценку применимости приближений (32), (33) и (35) дает табл. 2, где приведены восстановленная толщина слоя и соответствующие минимальные значения целевой функции (44).

Последняя строка табл. 2 подтверждает корректность использования приближения (32), (33) в выполненной выше обработке экспериментальных данных. По мере уменьшения $D$ для приближения (32), (33) характерен рост значений $\Phi_{\min }$, что означает ухудшение согласия „экспериментальных“ и расчетных распределений (38). Тем не менее вплоть до $D=50 \mu \mathrm{m}$ данное приближение обеспечивает приемлемую точность определения параметров слоя. В частности, соответствующие графики восстановленных спектров $n_{f}(\lambda)$ и $k_{f}(\lambda)$ визуально неотличимы от кривых 1 и 2 на рис. 3, которые соответствуют „,точным“ спектрам $n_{f}(\lambda)$ и $k_{f}(\lambda)$. Этот результат имеет место при использовании обеих аппаратных функций (36) и (37). В то же время с точки зрения согласия „экспериментальных“ и расчетных спектров (38) приближение (32), (33) более адекватно гауссовой, чем ступенчатой аппаратной функции, о чем говорит сравнение значений $\Phi_{\min }$ во втором и шестом столбцах табл. 2. Это результат в среднем более быстрого спада модулей $\left|I_{\alpha}\left(\eta_{l}\right)\right|$ в процессе увеличения $D$ в случае (36) по сравнению со случаем (37). 

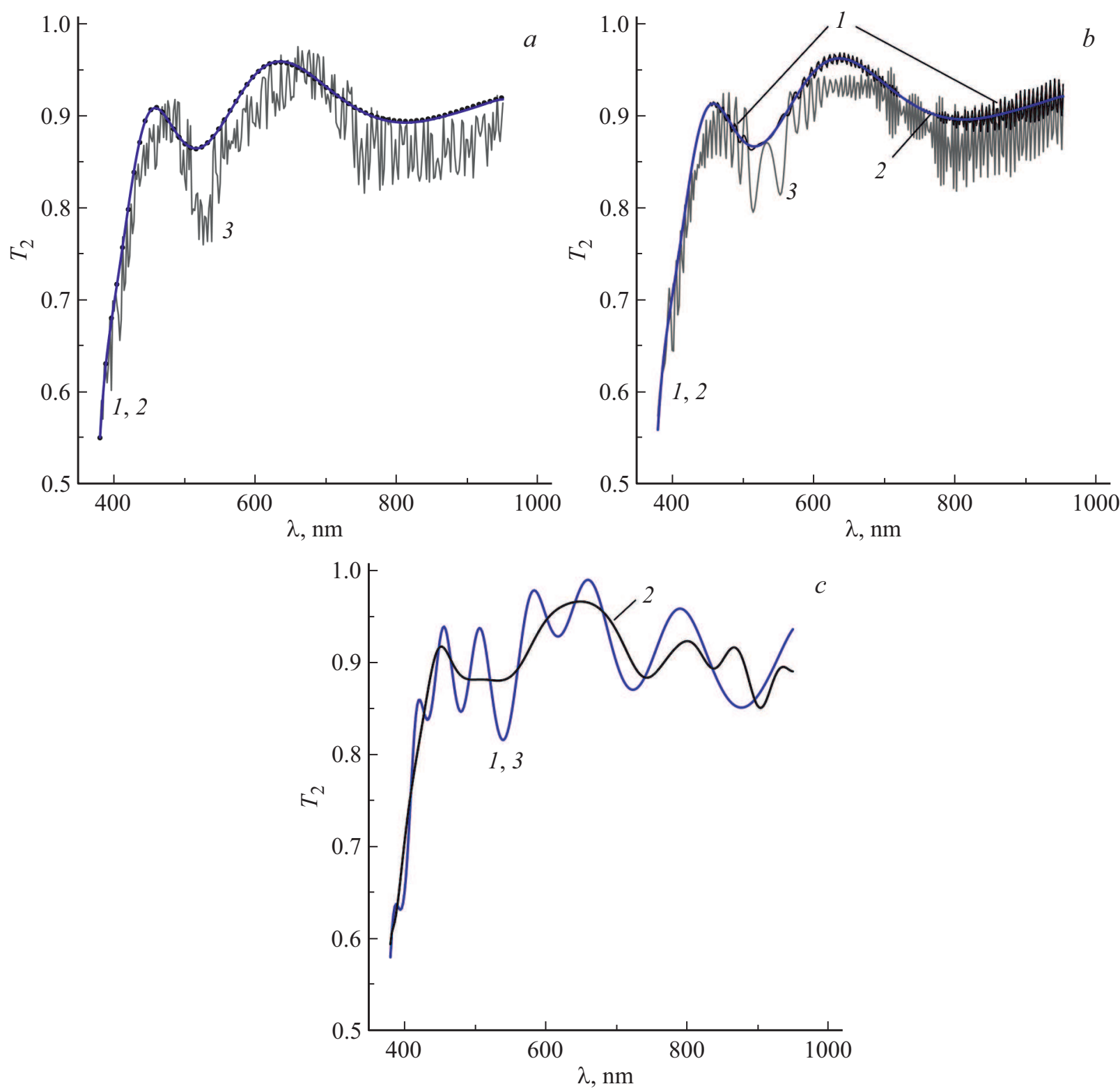

Рис. 4. Спектры $T_{2}(\theta, \lambda)$, рассчитанные на основании (9) для аппаратной функции (37) (кривые 1) и в приближениях (33) (кривая 2), (35) (кривая 3) после минимизации целевой функции (44) при $D=2.14 \mathrm{~mm}(a), D=50 \mu \mathrm{m}(b)$ и $D=1 \mu \mathrm{m}(c)$; дискретные точки - эксперимент.

В области $D \leq 10 \mu \mathrm{m}$ ошибки решения обратной задачи спектрофотометрии с использованием приближения (32), (33) резко возрастают. Об этом свидетельствуют данные табл. 2 и кривые 1 ' и 2 ' на рис. 3. Последние относятся к $D=10 \mu \mathrm{m}$ и аппаратной функции (36). Из табл. 2 видно, что при $D<5 \mu$ m предпочтительным при решении обратной задачи спектрофотометрии становится использование монохроматического приближения (35). В частности, при $D=1 \mu \mathrm{m}$ графики восстановленных в данном приближении функций $n_{f}(\lambda)$ и $k_{f}(\lambda)$ полностью совпали с кривыми 1 и 2 на рис. 3. Из табл. 2 видно, что приближение (35) более адекватно ступенчатой аппаратной функции (37), нежели гауссовой (38). Это объясняется сужением эффективных диапазонов интегрирования в (8), (9) при переходе от выражения (36) к (37).

Следует отметить, что табличные $d$, относящиеся к областям применимости приближений (32), (33) и (35), несколько превышают точное значение $d=355.29 \mathrm{~nm}$. Расчеты показали, что причиной этого расхождения является использование сравнительно большого значения $\Delta \lambda=2 \mathrm{~nm}$ при вычислении интегралов в (8) и (9). Переход к $\Delta \lambda=0.5 \mathrm{~nm}$ позволил названное расхождение устранить. Аналогичный результат имел место при коррекции данных вычислительных экспериментов методом подвижного окна [23] с сохранением $\Delta \lambda=2 \mathrm{~nm}$. 


\section{Заключение}

Выполнен анализ интегральных соотношений, описывающих отражательную $R_{\alpha}(\theta, \lambda)$ и пропускательную $T_{\alpha}(\theta, \lambda)$ способности структуры, состоящей из двух тонких слоев, находящихся на противоположных гранях плоскопараллельной подложки. Рассмотрено наклонное падение на структуру частично когерентного света. В случаях толстой (толщина $D \gg \lambda$ ) слабо поглощающей подложки, непрозрачной подложки и тонкой $(D \sim \lambda)$ подложки для функций $R_{\alpha}(\theta, \lambda)$ и $T_{\alpha}(\theta, \lambda)$ получены приближенные аналитические выражения (приближения толстой и тонкой подложек), не зависящие от аппаратной функции спектрофотометра. На их основе методом наименьших квадратов выполнена обработка экспериментальных данных для $R_{\alpha}(\theta, \lambda)$ и $T_{\alpha}(\theta, \lambda)$. Эксперимент выполнен в диапазоне от 380 до $950 \mathrm{~nm}$ для волн $s$ - и $p$-поляризации при двух углах падения света на структуру в виде слоя $\mathrm{ZnO}: \mathrm{Al}$, нанесенного магнетронным распылением мишени $\mathrm{ZnO}: \mathrm{Al}_{2} \mathrm{O}_{3}$ на стеклянную подложку. В результате определены спектры показателей преломления и поглощения подложки и слоя, а также толщина слоя. Дисперсионные характеристики слоя найдены путем их представления полиномом Лагранжа-Чебышева оптимальной степени без привлечения какой-либо физической дисперсионной модели. Установленные параметры структуры использованы для количественной оценки границ применимости аналитических приближений для $R_{\alpha}(\theta, \lambda)$ и $T_{\alpha}(\theta, \lambda)$ для гауссовой и ступенчатой моделей аппаратной функции спектрофотометра. Показано, что при полуширине аппаратных функций $\Delta \lambda=2 \mathrm{~nm}$ приближение толстой подложки эффективно, если $D \geq 50 \mu \mathrm{m}$, а приближение тонкой подложки - если $D \leq 5 \mu \mathrm{m}$. Вне этих диапазонов для решения обратной задачи спектрофотометрии необходимо применять интегральные формулы (8) и (9) для $R_{\alpha}(\theta, \lambda)$ и $T_{\alpha}(\theta, \lambda)$, которые требуют точного задания аппаратной функции спектрофотометра. Количественная реализация соответствующих алгоритмов требует дополнительных исследований.

\section{Финансирование работы}

Работа выполнена в рамках задания 1.3.03 „Разработка теории методов оптического контроля наноразмерных тонкопленочных структур“ ГПНИ „Фотоника, опто- и микро-электроника“, № госрегистрации 20161316.

\section{Конфликт интересов}

Авторы заявляют, что у них нет конфликта интересов.

\section{Список литературы}

[1] Vriens L., Rippens W. // Appl. Opt. 1983. V. 22. N 24. P. 4105. doi 10.1364/AO.22.004105

[2] Minkov D. // J. Opt. Soc. Am. A. 1991. V. 8. N 2. P. 306. doi 10.1364/JOSAA.8.000306
[3] Chen G., Tien C.L. // J. Heat Trans-T ASME. 1992. V. 114. N 3. P. 636. doi $10.1115 / 1.2911328$

[4] Richter K., Chen G., Tien C.L. // Opt. Engin. 1993. V. 32. N 8. P. 1897. doi 10.1117/12.147152

[5] Minkov D. // Appl. Opt. 1994. V. 33. N 33. P. 7698. doi 10.1364/AO.33.007698

[6] Anderson C.F., Bayazitoglut Y. // J. Thermophys Heat Tr. 1996. V. 10. N 1. P. 26. doi $10.2514 / 3.748$

[7] Gonzalez-Leal J.M., Prieto-Alcon R., Angel J.A., Minkov D.A., Marquez E. // Appl. Opt. 2002. V. 41. N 34. P. 7300. doi 10.1364/AO.41.007300

[8] Katsidis C.C., Siapkas D.I. // Appl. Opt. 2002. V. 41. N 19. P. 3978. doi 10.1364/AO.41.003978

[9] Centurioni E. // Appl. Opt. 2005. V. 44. N 35. P. 7532. doi 10.1364/AO.44.007532

[10] Fu K., Hsu P.F., Zhang Z.M. // Appl. Opt. 2006. V. 45. N 4. P. 653. doi 10.1364/AO.45.000653

[11] William E.V., Castro D. // Appl. Opt. 2007. V. 46. N 4. P. 502. doi 10.1364/AO.46.000502

[12] Domínguez-Gómez A.B., Mauricio-Sónchez R.A., MendozaGalván A. // Opt. Mater. 2018. V. 84. P. 564. doi 10.1016/j.optmat.2018.07.059

[13] Born M., Wolf E. Principles of Optics. Pergamon Press, 1970. 808 р.; Борн М., Воль $б$ Э. Основы оптики. М.: Наука, 1973. $719 \mathrm{c}$

[14] Анго A. Математика для электро- и радиоинженеров. М.: Наука, 1964. 772 c.

[15] Раутиан С.Г. // УФН. 1958. Т. 66. № 3. С. 475.

[16] Сотский А.Б., Кривецкий К.Н., Парашков С.О., Сотская Л.И. // ЖПС. 2016. Т. 83. № 5. С. 809; Sotsky A.B., Krivetskii K.N., Parashkov S.O., Sotskaya L.I. // J. Appl. Spectrosc. 2016. V. 83. N 5. P. 845. doi 10.1007/s10812-016-0373-3

[17] Смирнов В.И. Курс высшей математики. Т. 3. Ч. 2. М.: Наука, 1974. 672 с.

[18] Gao L., Lemarchand F., Lequime M. // Opt. Express. 2012. V. 20. N 14. P. 15735. doi 10.1364/OE.20.015734

[19] Стаськов Н.И., Сотский А.Б., Сотская Л.И., Филиппов В.В., Шулицкий Б.Г., Кашко И.А. // ЖПС. 2018. T. 85. № 4. C. 658; Staskov N.I., Sotsky A.B., Sotskaya L.I., Filippov V.V., Shulicky B.G., Kashko I.A. // J. Appl. Spectrosc. 2018. V. 85. N 4. P. 710. doi 10.1007/s10812-018-0709-2

[20] Korn G., Korn T. Mathematical Handbook for Scientists and Engineers. N. Y:: Mc Graw-Hill Book Company, 1968. 1130 р.; Корн Г., Корн Т. Справочник по математике для научных работников и инженеров. М.: Наука, 1973. 832 с.

[21] Hudson D.J. Statistics. Geneva, 1964. 450 p.; Худсон Д. Статистика для физиков. М.: Мир, 1970. 295 с.

[22] Сотский А.Б., Штейнгарт Л.М., Парашков С.О., Сотская Л.И. // Изв. РАН. Серия физическая. 2016. Т. 80. № 4. С. 465. doi 10.7868/S036767651604030X

[23] Сотский А.Б., Михеев С.С. // Вестник МГУ им. А.А. Кулешова. Серия В. 2019. № 2 (54). С. 49. 